n previous review articles in this series entitled "Opening up", John Gibbs gave a detailed account of interventional procedures performed to remove the obstacles often observed in congenital heart disease. ${ }^{12}$ The objective of this review is to describe in detail the more recent but equally useful techniques implemented in order to close and occlude congenital heart defects and vessel connections.

To begin with I would like to pay tribute to William Rashkind, Jim Lock, and Kurt Amplatz for their fundamental contributions to the progress achieved in this field.

\title{
PATENT DUCTUS ARTERIOSUS
}

Transcatheter closure of patent ductus arteriosus was the first "closing" interventional procedure to be performed routinely. This procedure constituted a significant step forward in the percutaneous treatment of congenital heart disease and was initially developed by Porstmann in the late 1970s. It was William Rashkind who pioneered this technique after designing ${ }^{3}$ the Rashkind PDA occluder which consists of a double umbrella of stainless steel and polyurethane foam, introduced through a long sheath from the pulmonary artery. ${ }^{3}$ The next method to be most widely implemented involved the use of coils. At first simple pushed coils were used with a relatively high rate of embolisation. When embolisation occurs, coils hindering flow in a pulmonary branch must be retrieved by means of a snare. Such a retrieval manoeuvre can be long and difficult.

Then gradual technical improvements, and especially the development of detachable coils, allowed closure of large arterial ducts, using several coils if necessary. ${ }^{4}$ This technique is safe and particularly efficient in small ducts (fig lA). However, the most spectacular progress has been the duct occluder developed by Kurt Amplatz. ${ }^{5}$ Like the other occluding devices he developed, it is made of a mesh of Nitinol (memory metal, a nickel-titanium alloy). It can be pushed inside a relatively small catheter and recovers its normal shape outside, in the body (fig $1 \mathrm{~B}$ ). Indications for closure of arterial ducts have become very broad as the technique is considered easy and safe. The most obvious indication is the presence of a large duct with a high flow rate. In such cases the main issue is the actual size of the patient. Indeed, in children $<5 \mathrm{~kg}$, in whom closure is mostly required and especially in premature babies, implantation of any device is difficult or impossible. Because of the small size of the aortic ampulla and pulmonary arteries, there is a risk that the device used, whether coils or Amplatzer PDA occluder, may protrude either into the left pulmonary artery or into the aorta. Embolisation is less likely to occur with this device than with coils. Retrieval from the pulmonary artery is difficult because of its shape and requires use of specific instruments such as biopsy forceps. Apart from cases of large left to right shunting, the most frequent indication for closure of small ducts is the anticipated risk, though very low, of endocarditis. In such instances, most teams generally perform the closure using coils. It is important to note that the cost of devices such as the Amplatzer PDA occluder is higher than that of simple coils.

\section{ATRIAL SEPTAL DEFECTS}

William Rashkind was a pioneer in developing the technique of atrial septal defect (ASD) closure in the late 1970s. On the basis of the Rashkind PDA Occluder, Jim Lock developed the famous Clamshell in 1989. Around the same period, Sideris initiated clinical trials with the Buttoned device. Other devices have been developed such as the Das Angel Wing, and the Babic AsDos. Finally, over the past few years, four major devices have become available: CardioSEAL ${ }^{6}$ and STARFlex, ${ }^{7}$ derived from the Clamshell with Dacron patches (with centering springs in the latter); the Amplatzer Septal Occluder (Fig 2B) made from Nitinol wire mesh like the arterial duct occluder described earlier, ${ }^{8}$ with a centering waist; the Helex device, ${ }^{9}$ made of a very thin PTFE curtain; and finally the latest generation Sideris patch without sutures or metallic frame. ${ }^{10}$ Among the various types of atrial septal defects, some are not currently amenable to percutaneous closure such as ostium primum and superior or inferior sinus venosus defects. Indeed, the margins of the 


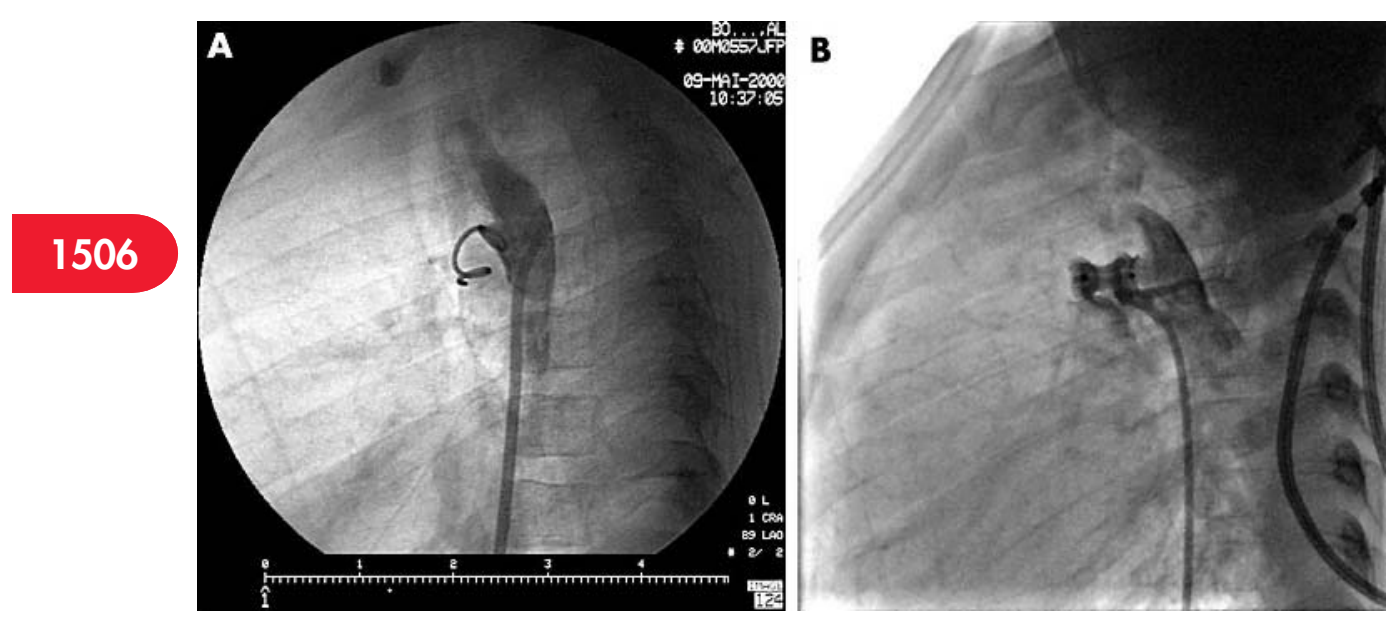

Figure 1 Patent ductus closure. (A) Small ductus closed with a single coil. (B) Larger ductus with an Amplatzer PDA device. Note the immediate residual flow. It will close within a few hours.

orifice must be wide enough to accommodate the edges of a closing device. Only the ostium secundum (OS) and patent foramen ovale (PFO) type defects can be successfully closed via the percutaneous approach.

Percutaneous closure of an ostium secundum type defect has become routine procedure. Indication for closure is the clinical or echographic evidence of a significant left-to-right shunt causing dilatation of the right chambers. In such instances, the defect is generally wide, but it may also be relatively small in some cases. The simplicity and ease of this procedure have led certain teams to widen their indications to small size defects. Diagnosis is made on the basis of transthoracic echocardiography, and device implantation must be performed under transoesophageal echocardiography or even intracardiac echocardiography guidance, sometimes with the help of three dimensional reconstruction. The static diameter of the defect is assessed by transoesophageal echo first, and then the stretched diameter, measured with a sizing balloon, is used to select the proper diameter of the device. The same technique is used for any device. So far, the Amplatzer septal occluder is currently the most widely used device because it is easy to implant and allows closure of large orifices with an excellent success rate in the majority of cases. ${ }^{11}$ STARFlex is used for defects up to $20 \mathrm{~mm} .{ }^{7}$ Instances of device arm fracture have been observed with STARFlex. Closure of multiple defects is possible when they are close to one another, or by using several occluders when they are farther apart (fig 2A). Fenestrated defects consisting

\section{Percutaneous closure of the patent ductus} arteriosus

- Is the treatment of choice

- It is not always possible in very small neonates, especially in premature babies

- Procedural success can be achieved in most cases using the appropriate device

- Coils are preferably used in small ducts and more expandable prostheses in larger ducts

- Risks associated with the procedure are embolisation of the device or protrusion into the aorta or pulmonary artery

- This procedure is considered safer than surgical closure in adults in whom the arterial duct is often calcified of several small orifices can be occluded with specific prostheses.

Device implantation is sometimes difficult, mainly due to the septum orientation. Many technical solutions have been proposed. ${ }^{12}$ Some devices are easy to withdraw and to reposition, such as the Amplatzer and the Helex. Complications may occur during and after the procedure. ${ }^{13}$ Air or clot embolism caused by the long and large sheath used may be avoided using a precise protocol and coagulation monitoring. With experience, device embolisation has become a rare occurrence which happens as a result of inadequate sizing of the defect or incorrect device placement. Retrieval is sometimes possible using large sheaths and snares, but it is not always easy and the surgical approach is sometimes necessary. Finally, a few cases of cardiac perforation have been reported with various devices. Consequently, a close collaboration with the echocardiographer is necessary for precise assessment of the defect and for device implantation. Various adverse events may occur such as rhythm disturbance or migraine. They usually rapidly improve. Extremely rare cases of clot formation on the device have been reported despite appropriate antiplatelet treatment.

The PFO is a near to normal anatomy of the inter-atrial septum. Indeed, strictly speaking the PFO cannot be considered a defect as it is frequent in the normal population. Diagnosis is based upon evidence of a right-to-left shunt, permanent or not. The technique used is contrast echocardiography with intravenous injection of a solution containing micro air bubbles or other micro particles. The atrial septum is observed under transthoracic or transoesophageal echocardiographic guidance for passage of the micro bubbles into the right atrium, either spontaneously or induced by cough or Valsalva manoeuvre. Paradoxical embolism or in situ clot creation and embolisation may induce clot embolism in any territory, causing a stroke or a simple transient ischaemic attack, or various embolic events. But closure of the PFO for this indication remains subject to controversy. ${ }^{14}$ It may also be responsible for the platypnoea-orthodeoxia syndrome. ${ }^{15}$ Pneumonectomy, phrenic paralysis, or aortic root enlargement may alter the interaction of the organs in the chest and induce reopening of the PFO with right to left shunting. In this setting, transcatheter closure is clearly indicated as it is often immediately efficient. Recently, a dramatic reduction or even disappearance of previously reported migraine has been observed in patients undergoing PFO closure for other 

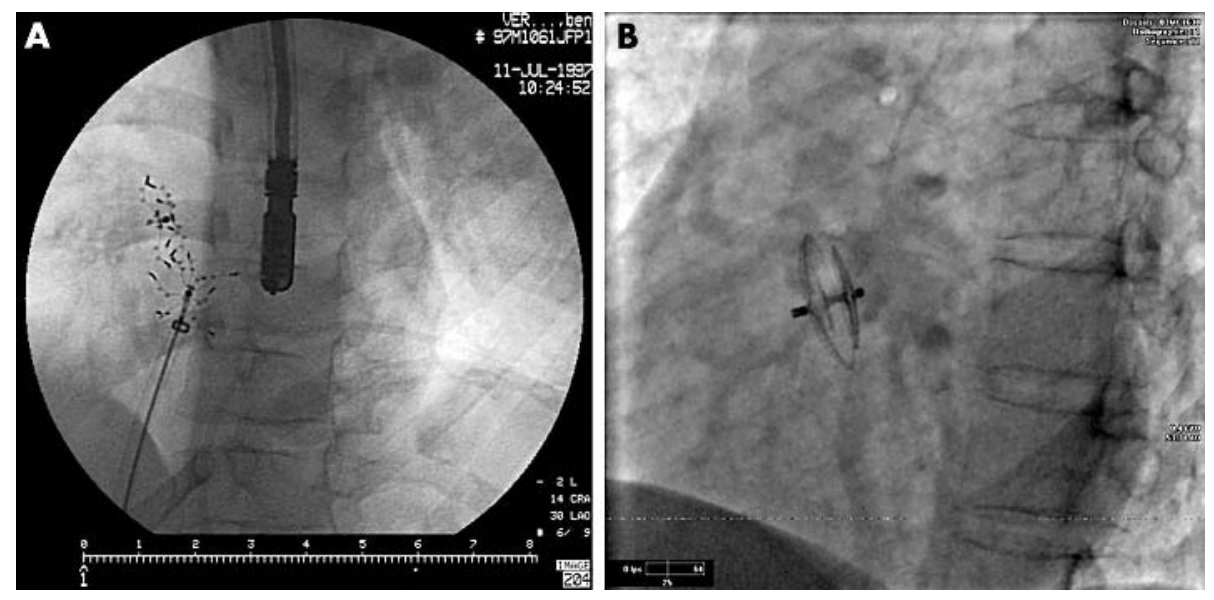

Figure 2 Atrial septal defect (ASD) closure. (A) Amplatzer ASD device in place. (B) Two separate defects closed with two CardioSEAL devices.

indications. ${ }^{16}$ Furthermore, studies have shown a higher prevalence of PFO in patients experiencing migraine with aura compared with the general population. Studies on this indication are being initiated. Though the exact mechanism has not been unravelled yet, passage of a substance through the PFO, while being metabolised in the lung, could provide an explanation.

\section{VENTRICULAR SEPTAL DEFECTS}

Closure of ventricular septum defects (VSDs) presents a number of important difficulties, to a greater extent than ASD closure. Jim Lock performed the first procedures. ${ }^{17}$ These communications can be located in the perimembranous septum next to the aortic and/or pulmonary valve. They may also be found in the muscular part of the ventricular septum: atrioventricular, trabecular or apical septum, or at the junction between the septum and the ventricular walls on the diaphragmatic as well as the anteroposterior side. All but the atrioventricular defect or defects involving the valves or their subvalvar apparatus are potentially accessible to percutaneous closure. The defect must be crossed either via the venous approach from the right ventricle, or, in most cases, via the arterial approach from the left ventricle engaged through the aortic or the mitral valve. For positioning the long sheath through which the device is pushed, a circuit must be formed with a guidewire across the VSD from the femoral or jugular vein and the femoral artery.

Various devices can be used. Currently, the muscular VSD Amplatzer occluder is well adapted to VSDs located in the muscular part of the septum. ${ }^{18}$ PDA Amplatzer devices can be used for apical VSDs or those at the junction with the

\section{Percutaneous closure of atrial septal defects}

- At present, only ostium secundum and patent foramen ovale type atrial septal defects are amenable to transcatheter closure

- About $60 \%$ of ostium secundum defects can be treated percutaneously, including certain types of multiple defects

- This is a very safe technique allowing complete closure in the majority of cases. Only a few cases of untoward side effects have been reported such as clot formation on the device, induced rhythm disturbance, late device embolisation, or heart perforation

- Despite the role of patent foramen ovale in various conditions, its closure remains controversial ventricular walls by positioning their single disk on the left side of the septum. A specific prosthesis was recently developed for perimembranous VSDs. ${ }^{19}$ As the device must not hinder the motion of the aortic valve leaflets, it was designed with an asymmetric shape. A specific delivery catheter is used to orientate the device correctly. Even closure of multiple defects is feasible (fig 3). Once the device has been implanted, embolism is a rare occurrence and complete closure can be rapidly achieved in most cases. At present, the most frequent VSDs, namely the perimembranous type, are just becoming amenable to this technique. Conversely, muscular VSDs which cannot be easily treated by the surgical approach are good indications for the percutaneous approach.

The results of the few VSD closure procedures performed so far are very encouraging, but larger series are warranted, and one must remember that this procedure is often difficult to perform. Though surgical results are excellent, the associated morbidity rate is probably higher. Surgical approach to muscular, especially apical, VSDs may be difficult and percutaneous procedures offer a clear benefit. Combined procedures, including transcatheter muscular VSD closure and surgical repair of the other abnormalities, have been performed.

\section{PARAVALVAR LEAKS}

In certain relatively frequent though seldom treated cases, a paravalvar leak is observed. Though transcatheter closure can be attempted, it remains nevertheless one of the most difficult interventions of its kind, if not the most difficult. Appropriate assessment of the leak can only be performed by transoesophageal echocardiography. Small jets cause severe haemolysis, and large leaks result in cardiac insufficiency. In some instances, the defect may involve more than half of the valve circumference. As far as the mitral valve is concerned, implantation of a device is carried out by means of an arteriovenous loop formed through the atrial septum following transseptal puncture. ${ }^{20}$ The results achieved so far have been rather disappointing, mainly for lack of an appropriate device with an adapted shape. Crossing the leak is easier at the aortic valve level, and, as it is often small, the results are better.

\section{VASCULAR EMBOLISATION}

The principle of embolisation consists of positioning a device at the origin or at the distal extremity of the vessel in order to achieve complete or partial mechanical occlusion. Thrombotic 

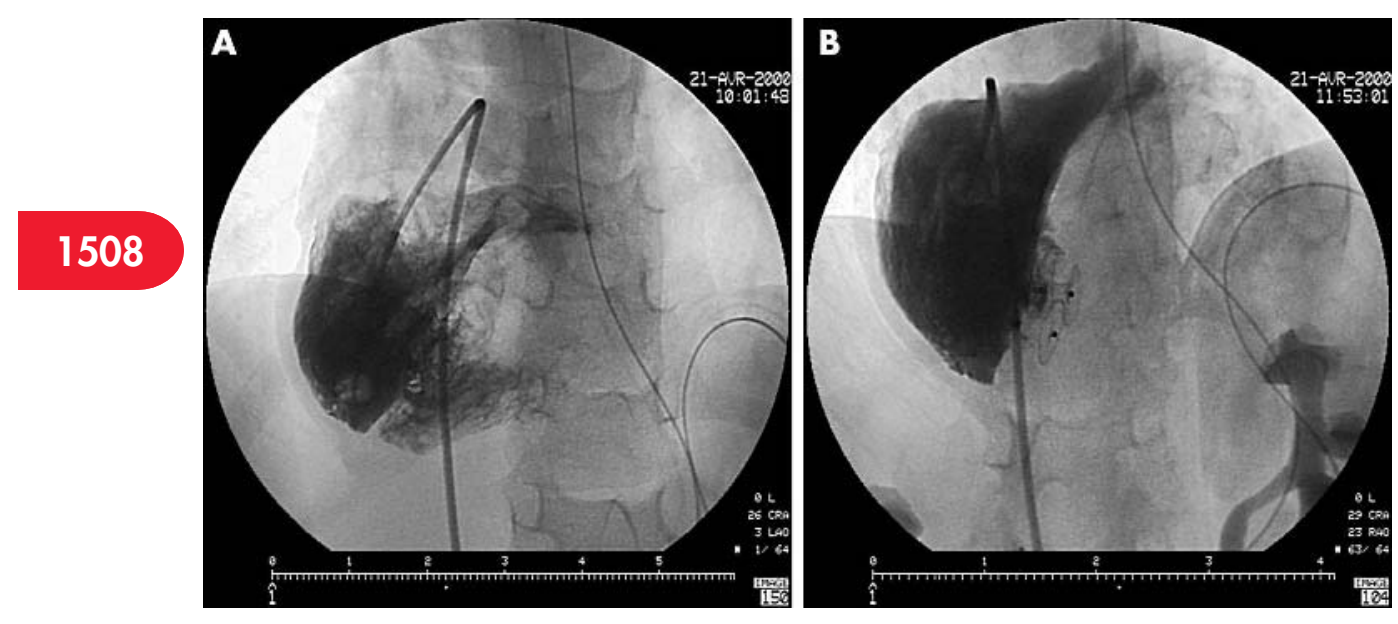

Figure 3 Multiple ventricular septal defect (VSD) closure. (A) Complex heart disease with "Swiss cheese" VSD. (B) Quite complete closure with two Amplatzer VSD devices.

occlusion of the vessel is brought about by slowing down the blood flow and by the device characteristics. Various devices are available.

\section{Detachable balloons}

These are deflated balloons inserted through a guiding catheter and pushed to the implantation site. They are inflated by means of a micro-catheter containing a contrast solution. Upon withdrawal of the micro-catheter, the balloon is closed by a valve.

This complex method requires relatively large catheters, and the balloons used have been reported to deflate prematurely. Detachable balloons are best used in medium sized vessels.

\section{Coils}

These are metallic wires of variable shape, length, and rigidity. They can be either bare or with strands and can be either pushed and delivered or detachable. Implantation of detachable coils can be controlled and delivery is performed only when the positioning is judged acceptable. Various mechanisms for detachment have been developed.

\section{Prostheses}

Any device initially designed for occlusion of arterial ducts and atrial or ventricular septal defects can be used. These types of devices include the CardioSEAL/STARFlex and Amplatzer devices.

In addition, the new Amplatzer "Plug" made of very thin Nitinol wire mesh and very easy to position is worth mentioning.

\section{Indications}

Coronary artery fistulae

Treatment of fistulae between the coronary artery and the cardiac chambers has been carried out via the surgical

\section{Percutaneous closure of ventricular septal}

defects

- These procedures are not performed frequently because of technical difficulties

- Only mid-muscular, apical, and perimembranous VSDs can be treated percutaneously

- As some VSDs are difficult to close surgically, apical or "Swiss cheese" transcatheter techniques are promising approach for many years. Such fistulae may involve the right or the left coronary artery and sometimes both. The fistula may drain into any of the cardiac chambers though most often into the right chambers. Accurate diagnosis of the lesion by means of selective contrast injection into each coronary artery using conventional angiographic views is essential. The normal coronary branches must be carefully studied to ensure that they are not occluded and potential multiple feeding to the fistula must be identified. The fistula flow, which is often very significant, may hinder visibility of the native coronary flow. It is crucial that vital coronary arteries should not be occluded by the procedure; contrast injections should be made (if necessary beyond a balloon inflated in the main vessel) in order to visualise all branches while looking for ECG changes. The feeding coronary artery may have a large diameter allowing easy advancement of the closure equipment to the fistula. Its opening into the cardiac chamber may be very large or very narrow. Successive narrowings may be observed. Except for the very small ones, these fistulae do not close or become reduced of their own accord. The occlusion method (coils or occluding device) is selected according to the angiographic aspects (fig 4). Only a few teams have had the opportunity to perform such procedures because of the relative rarity of this pathology. ${ }^{21}$ The indication may be the presence of significant flow in the fistula, creating a left-to-right shunt or less often a left-to-left shunt, and also cardiac insufficiency caused by coronary steal.

\section{Surgical systemic pulmonary shunts}

Most surgical shunts are amenable to transcatheter closure. Indication for closure may be the presence of excessive flow or the fact that the shunt has become superfluous. Transcatheter closure may also be performed in some specific situations at the time of complete repair, just before surgery, due to its simplicity and to the potential surgical difficulties. Devices used include pushed or detachable coils inserted via the arterial approach, especially for Blalock-Taussig shunts. As coil migration is not a rare occurrence, closure can be performed by placing a balloon in the pulmonary branch in order to stop the flow. Potts or Waterston shunts closure should only be performed, if pulmonary resistance allows it, with double-disk devices such as the CardioSEAL/STARFlex device or PDA, ASD, or VSD Amplatz occluders. 

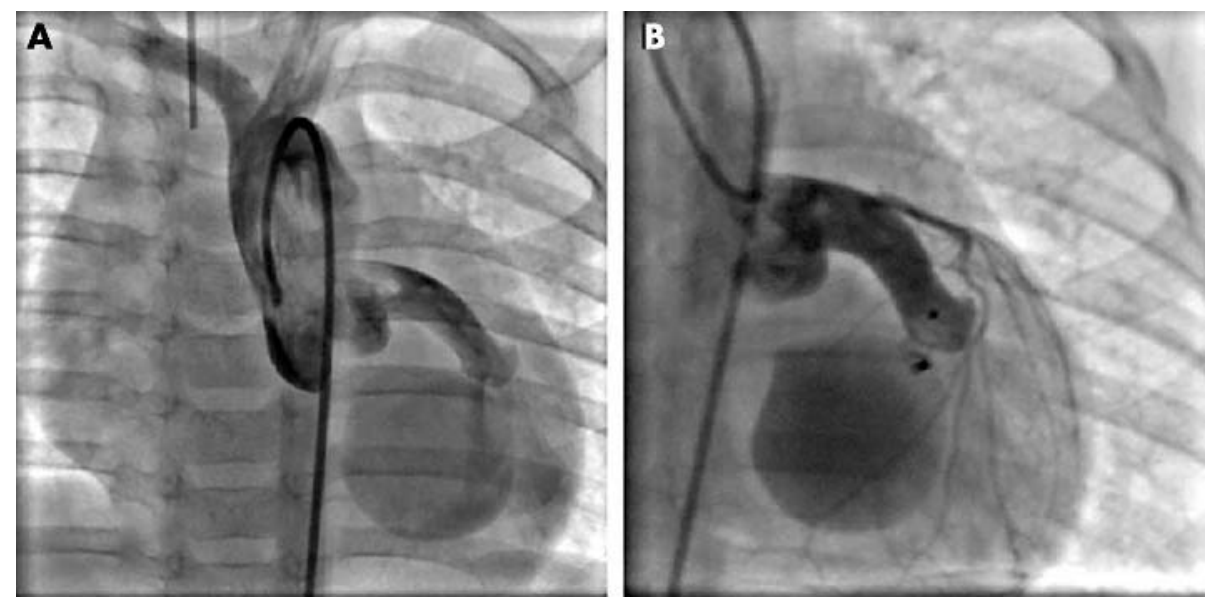

Figure 4 (A) Coronary artery fistula from circumflex to coronary sinus. (B) Closure by implantation of a small Amplatzer PDA device implanted from the right side.

\section{Aortopulmonary collateral vessels}

It is necessary to identify clearly which vessels should be occluded and when. These major aortopulmonary collateral arteries (MAPCAs), originating in the aorta or its branches and feeding pulmonary segments, may or may not be connected to the central pulmonary artery. Prior diagnostic catheterisation plays a crucial role by allowing visualisation of the central pulmonary artery through direct or indirect contrast injection (aortography, MAPCA, or venous pulmonary wedge injections). Selective injections in MAPCAs visualise those connected to the central pulmonary artery and amenable to closure, though the other collateral vessels should be unifocalised or surgically connected to the central pulmonary artery. Transcatheter closure can be performed before the complete repair operation. Indeed, the considerable flow of the MAPCAs results in a very significant venous pulmonary return which may hinder the performance of surgery. Closure can also be undertaken after the operation, in particular after "unifocalisation". Transcatheter closure can be carried out with various devices described earlier, and especially with coils or Amplatzer occluding devices.

Device selection is made according to size, anatomy, and presence of tapering segments. The arterial approach is used; however, when the procedure is performed before complete surgical repair, antegrade closure can be achieved by crossing the ventricular septal communication. Approach selection should be made according to the implantation angle of the vessels.

\section{Pulmonary sequestrations}

Sequestrations are vascularised pulmonary territories without bronchi for ventilation. Due to the flow volume and risk of infection, occlusion is often considered. This is typically observed in patients with scimitar syndrome. In most cases, occlusion can be performed with pushed coils; however, the size of the feeding artery may require use of an occluding device.

\section{Pulmonary arteriovenous fistulae}

Fistulae between the pulmonary artery and the pulmonary vein are of congenital origin in most cases, they are often single, and voluminous. They cause cyanosis and in some instances embolic accidents. ${ }^{22}$ Acquired fistulae such as those observed at long term follow up after a surgically created cavopulmonary shunt are mostly small and multiple. All kinds of devices, and especially coils, can be used.

\section{Left superior vena cava}

The persistent superior left vena cava generally drains into the right atrium through the coronary sinus, and the innominate vein is often absent. Conversely, the left superior vena cava may drain into the left atrium, directly or through an unroofed coronary sinus. In this setting, closure of the vena cava is recommended because of the resulting right-toleft shunt. Occlusion can be performed percutaneously, especially via the left jugular approach with occluding devices rather than coils, placed just above the heart. In the presence of multiple perforations in the coronary sinus, occlusion of the vena cava may paradoxically induce left to right shunting. In such instances, even if transcatheter perforation closure may be considered, surgical management is probably the most appropriate option.

\section{Superior vena cava to inferior vena cava or pulmonary vein shunts}

Following the performance of a superior cavopulmonary connection, pulmonary resistance may become significant. In such settings, shunts to vessels with less resistance can appear. In most cases these connections drain into the inferior vena cava, but may also connect with the pulmonary veins. They arise from normal vessels, such as the azygos vein if not ligated during surgery, or a forgotten left superior vena cava draining into the coronary sinus, or other natural vessels which become enlarged. When these shunts are large and not numerous, closure can be performed with several kinds of occluding devices. Though occlusion of multiple, small size vessels can be successfully performed, other new collateral connections appear.

\section{POTENTIAL COMPLICATIONS}

Potential risks associated with these interventional procedures have always been considered as higher than those of previously used techniques. These risks are especially related

\section{Vessel embolisation}

- Various native vessels or surgically created ones may be closed by transcatheter technique

- In some instances, such closures have to be performed just before or after another surgical procedure

- Various devices can be used depending on the length size, and shape of the vessels 
to the use of voluminous and complex devices through large lumen catheters. However, the theoretical risks should not be overestimated compared to the risks of surgical treatment.

These procedures can generate immediate and acute complications due to device embolisation induced by incorrect placement or inaccurate assessment of the orifice

or the vessel size. Such a potential occurrence should always be anticipated, and consequently the operator should be familiar with the retrieval techniques and ensure that the equipment required for such manoeuvres is readily available, or arrange for easy access to surgical facilities. The retrieval equipment consists of large size guiding catheters and various snares or forceps.

However, such a dramatic complication is neither the most frequent nor the most difficult to deal with. There is another potential and much more hazardous complication, namely air or clot embolism caused by inadequate anticoagulation. Appropriate heparin treatment is essential to prevent its occurrence. It can be treated in most cases with general or local fibrinolysis.

Finally, mid to long term complications have been reported. Instances of device fracture have been described, generally without clinical consequences, as well as rare cases of cardiac embolism and perforations. Persistence of a residual shunt has caused haemolysis in some cases.

\section{CONCLUSION}

Transcatheter treatment of congenital heart defects has been considerably enhanced thanks to the pioneers of the technique and to very gifted and inventive operators.

Let us not forget the crucial part played by William Rashkind, Jim Lock, Kurt Amplatz and many others. These improvements have been made in spite of the limited number of patients, their size, and frequent procedural difficulties. The purpose of these techniques is to avoid surgery provided that they can prove to be as safe and efficient.

Transcatheter management of complex defects such as perimembranous VSDs, completion of partial cavopulmonary connection, and full treatment of tetralogy of Fallot is gradually becoming possible. In such a complex field, we should remain both ambitious and realistic and keep all medical and surgical options open. Cooperation between the operators in a medicosurgical unit is a necessary condition.

\section{REFERENCES}

1 Gibbs JL. Interventional catheterisation. Opening up I: the ventricular outflow tracts and great arteries. Heart 2000;83:111-15.

2 Gibbs JL. Interventional catheterisation. Opening up II: venous return, the atrial septum, the arterial duct, aortopulmonary shunts, and aortopulmonary collaterals, Heart 2000;83:237-40.

3 Rashkind WJ, Mullins CE, Hellenbrand WE, et al. Nonsurgical closure of patent ductus arteriosus: clinical application of the Rashkind PDA occluder system. Circulation 1987;75:583-92.

4 Hijazi ZM, Geggel RL. Transcatheter closure of large patent ductus arteriosus (> or $=4 \mathrm{~mm}$ ) with multiple Gianturco coils: immediate and mid-term results. Heart 1996;76:536-40

5 Masura J, Walsh KP, Thanopoulous B, et al. Catheter closure of moderate- to large-sized patent ductus arteriosus using the new Amplatzer duct occluder: immediate and short-term results. J Am Coll Cardiol 1998;31:878-82.

- First clinical study of the new PDA Amplatzer device, involving 24 patients with a relatively large ductus. Complete closure was obtained in one third of the cases, and trivial to small shunts in the others. At 24 hours Doppler revealed complete closure in all. This initial study shows the true efficacy of this new device.

6 Carminati M, Giusti S, Hausdorf G, et al. A European multicentric experience using the CardioSeal and Starflex double umbrella devices to close interatrial communications holes within the oval fossa. Cardiol Young 2000;10:519-26.

- This paper presents the only published results of a multicentre study using the CardioSEAL and STARFlex devices in a series of 117 cases. Apart from device embolisation the main issue was device arm fracture which occurred in large devices, but without related complications.

7 Carminati M, Chessa M, Butera G, et al. Transcatheter closure of atrial septal defects with the STARFlex device: early results and follow-up. J Interv Cardiol $2001 ; 14: 319-24$

8 Masura J, Gavora P, Formanek A, et al. Transcatheter closure of secundum atrial septal defects using the new self-centering Amplatzer septal occluder: initial human experience. Cathet Cardiovasc Diagn 1997;42:388-93.

- Initial human experience with the new Amplatzer septal occluder. This study involved 30 patients including adults. The mean stretched diameter of the ostium secundum atrial defect was $14 \mathrm{~mm}$ (7-19 mm). The device was always well implanted with complete closure in 17 cases and trivial shunt in 10. There were no complications during the procedure and follow up. This study marked the beginning of the Amplatzer septal occluder story.

9 Zahn EM, Wilson N, Cutright W, et al. Development and testing of the Helex septal occluder, a new expanded polytetrafluoroethylene atrial septal defect occlusion system. Circulation 2001;104:711-6.

10 Sideris EB, Sideris CE, Stamatelopoulos SF, et al. Transcatheter patch occlusion of experimental atrial septal defects. Catheter Cardiovasc Interv 2002:57:404-7.

11 Omeish A, Hijazi ZM. Transcatheter closure of atrial septal defects in children \& adults using the Amplatzer septal occluder. J Interv Cardiol $2001 ; 14: 37-44$

12 Harper RW, Mottram PM, McGaw DJ. Closure of secundum atrial septal defects with the Amplatzer septal occluder device: techniques and problems. Catheter Cardiovasc Interv 2002;57:508-24.

- Extensive paper on ASD closure using the Amplatzer device. Indications, technical aspects of a normal procedure, including sizing, echocardiographic monitoring, and deployment of the devices, are described. Specific problems of patent foramen ovale are reviewed.

13 Chessa M, Carminati M, Butera G, et al. Early and late complications associated with transcatheter occlusion of secundum atrial septal defect. J Am Coll Cardiol 2002;39:1061-5.

- This group in Milan has a wealth of experience in ASD closure and has extensively published its results. This paper summarises the complications in a cohort of 417 cases of implantation using the two major ASD devices, and concludes that the procedure is safe.

14 Meier B, Lock JE. Contemporary management of patent foramen ovale. Circulation 2003;107:5-9.

- This paper summarises the clinical implications of the patent foramen ovale and the indications for closure, with many important references quoted.

15 Piechaud JF. Hypoxemia related to right-to-left shunting through a patent foramen ovale: successful percutaneous treatment with the CardioSeal device. J Interv Cardiol 2001; 14:57-60.

16 Morandi E, Anzola GP, Angeli S, et al. Transcatheter closure of patent foramen ovale: a new migraine treatment? J Interv Cardiol 2003;16:39-42.

17 Lock JE, Block PC, McKay RG, et al. Transcatheter closure of ventricular septal defects. Circulation 1988;78:361-8.

18 Thanopoulos BD, Tsaousis GS, Konstadopoulou GN, et al. Transcatheter closure of muscular ventricular septal defects with the Amplatzer ventricula septal defect occluder: initial clinical applications in children. J Am Coll Cardiol 1999:33:1395-9.

- The muscular VSD closure with a new Amplatzer device in a small initial number of cases. Successful implantation and complete closure was achieved in all cases, and the only complication reported was a transient left bundle branch block in two of six cases.

19 Hijazi ZM, Hakim F, Haweleh AA, et al. Catheter closure of perimembranous ventricular septal defects using the new Amplatzer membranous VSD occluder: initial clinical experience. Catheter Cardiovasc Interv 2002;56:508-15.

20 Piechaud JF. Percutaneous closure of mitral paravalvular leak. J Interv Cardiol 2003; 16:153-5

- Description of the technical aspects of the closure of paravalvular leak in the mitral position. No clinical data are presented.

21 Armsby LR, Keane JF, Sherwood MC, et al. Management of coronary artery fistulae. Patient selection and results of transcatheter closure. J Am Coll Cardiol 2002;39:1026-32

22 Chessa $M$, Drago $M$, Krantunkov $P$, et al. Differential diagnosis between patent foramen ovale and pulmonary arteriovenous fistula in two patients with previous cryptogenic stroke caused by presumed paradoxical embolism. J Am Soc Echocardiogr 2002;15:845-6. 